\title{
Optimization of storage conditions when banking autologous split-thickness skin grafts
}

\author{
Cassandra Edlund1', Alexandra Karström¹, Jian Fransén ${ }^{2,3}$, Fredrik Huss ${ }^{2,3}$ \\ ${ }^{1}$ Tissue Establishment, Department of Plastic and Maxillofacial Surgery, Uppsala University Hospital, Uppsala, Sweden \\ ${ }^{2}$ Burn Centre, Department of Plastic and Maxillofacial Surgery, Uppsala University Hospital, Uppsala, Sweden \\ ${ }^{3}$ Department of Surgical Science, Plastic Surgery, Uppsala University, Uppsala, Sweden
}

\begin{abstract}
Background
Autologous split-thickness skin grafts (STSG) is the gold standard in treating burn injuries. Storage of STSGs is sometimes necessary and is a routine method in burn care with acceptable results.

Numerous different nutritional media are used around the world, at our burn centre we use RPMI 1640 media. It is well known that there is a successive deterioration of viability, and thus graft take, with storage time paralleled by a transition from viable grafts to more of a biological dressing. More or less arbitrarily the maximum storage time is considered to be approximately 4 weeks. The aim of this study is to explore an optimal storage medium yielding the highest number of viable cells at the time of retransplantation.

In the first step we examined ten different nutritional media used for banking of autologous STSGs by evaluating cell viability over time using cultured human keratinocytes from healthy donors.
\end{abstract}

\section{Results}

The viability of the cells at day 0 was regarded as $100 \%$. Average viability of the keratinocytes gradually declines over time. Figure 1 illustrates the summarized data of percent viable cells from day 1 to day 30 in storage. The summarized data at day 30 for all five substudies, figure 2, shows that there were two nutritional fluids with $10 \%$ or more viable cells at day 30 (McCoy's $5 \mathrm{~A}$ medium and $\mathrm{NaCl}$ $+5 \%$ glucose). Four nutritional media had 5-9\% viable cells and four nutritional media less than $5 \%$ viable cells at day 30 .

The study was repeated five times.

Three nutritional media had zero viable cells at day 30 in storage ( $\mathrm{NaCl}$, Leibovitz's L-15 medium and Ham's F12 nutrient mix). They were therefore excluded after the fourth repetition of the procedure and will not be included in further studies.

Figure 2, Cell viability at day 30 of storage

\begin{tabular}{|l|l|l|}
\hline Ranking & Nutritional fluid & \% Cell viability at day 30 \\
\hline 1 & McCoy's 5A medium & 12,3 \\
\hline 2 & $\mathrm{NaCl}+5 \%$ glucose & 10,9 \\
\hline 3 & RPMI 1640 medium & 7,83 \\
\hline 4 & DMEM + 5\% FCS & 7,40 \\
\hline 5 & DMEM + 2\% FCS & 6,62 \\
\hline 6 & Lirwalls medium* & 5,30 \\
\hline 7 & DMEM & 1,11 \\
\hline 8 & NaCl & 0 \\
\hline 9 & Leibovitz's L-15 medium & 0 \\
\hline 10 & Ham's F12 nutrient mix & 0 \\
\hline
\end{tabular}

\begin{abstract}
Methods
Ten nutritional media were selected based on in vitro use, clinical use, or for theoretical reasons.

Human skin was obtained from 4 healthy donors undergoing plastic surgery at Uppsala University Hospital. Keratinocytes were cultured to subconfluence using serum-free cell culturing media to the required amount of cells. $1^{*} 10^{6}$ cells $/ \mathrm{mL}$ were diluted in various nutritional media and stored at $4-7^{\circ} \mathrm{C}$ for up to 30 days.

Sampling was done at day $1,4,8,14,21$, and 30 in storage. Evaluation of cells included manual cell count in Bürker chamber, viability measuring with trypan blue, visual check for cell aggregates and ability to resolve into suspension.
\end{abstract}

Figure 1 Cell viability over time

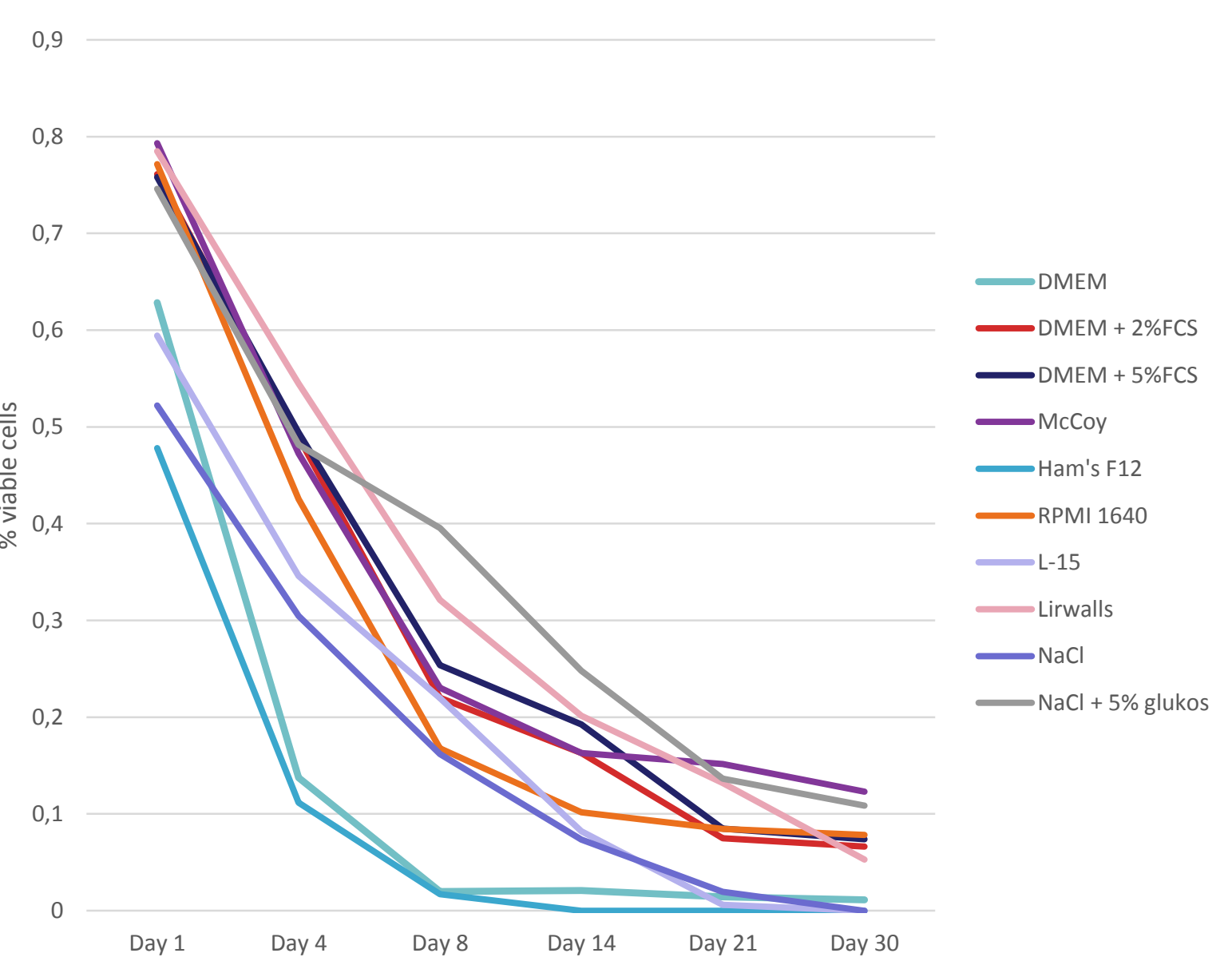

\section{Discussion}

Over all, as anticipated, quite few viable cells were seen after 30 days of storage in all nutritional media. Mutual for all nutritional media are a rapid decline in cell viability during day 1-8, ensuing a reduced declining rate regarding viability. Days of storage is therefore a central parameter in the decision of which nutritional media to use.

Since the aim of this study is to further improve an optimal storage media and to prolong the time viable STSGs can be stored with maintained or increased take rate, the study will continue with further studies on both cultured keratinocytes and human STSGs. 